\title{
Weak Microbial Metabolites: a Treasure Trove for Using Biomimicry to Discover and Optimize Drugs
}

\author{
DZdenek Dvorak, ${ }^{1}$ Max Klapholz, ${ }^{1}$ Thomas P. Burris, Benjamin P. Willing, Antimo Gioiello, \\ Roberto Pellicciari, Francesco Galli, John March, Stephen J. O’Keefe, R. Balfour Sartor, \\ Chang H. Kim, Maayan Levy, and Sridhar Mani \\ Department of Cell Biology and Genetics, Palacký University, Olomouc, Czech Republic (Z.D.); Department of Microbiology, \\ University of Pennsylvania, Philadelphia, Pennsylvania (M.K., M.L.); The Center for Clinical Pharmacology, Washington University \\ in St. Louis and St. Louis College of Pharmacy, St. Louis, Missouri (T.P.B.); Department of Agricultural, Food and Nutritional \\ Science, University of Alberta, Edmonton, Alberta (B.P.W.); Department of Pharmaceutical Sciences, University of Perugia, \\ Perugia, Italy (A.G., F.G.); TES Pharma, Corso Vannucci, Perugia, Italy (R.P.); The Department of Biological and Environmental \\ Engineering, Cornell University, Ithaca, New York (J.M.); Division of Gastroenterology and Nutrition, UPMC Presbyterian \\ Hospital, Pittsburgh, Pennsylvania (S.J.O.); Division of Gastroenterology and Hepatology, Department of Medicine, Center for \\ Gastrointestinal Biology and Disease, University of North Carolina at Chapel Hill, Chapel Hill, North Carolina (R.B.S.); Department \\ of Pathology, Mary H. Weiser Food Allergy Center, and Rogel Cancer Center, University of Michigan School of Medicine, \\ Ann Arbor, Michigan (C.H.K.); and Department of Medicine, Albert Einstein College of Medicine, Bronx, New York (S.M.)
}

Received April 22, 2020; accepted July 22, 2020

\section{ABSTRACT}

For decades, traditional drug discovery has used natural product and synthetic chemistry approaches to generate libraries of compounds, with some ending as promising drug candidates. A complementary approach has been to adopt the concept of biomimicry of natural products and metabolites so as to improve multiple drug-like features of the parent molecule. In this effort, promiscuous and weak interactions between ligands and receptors are often ignored in a drug discovery process. In this Emerging Concepts article, we highlight microbial metabolite mimicry, whereby parent metabolites have weak interactions with their receptors that then have led to discrete examples of more potent and effective drug-like molecules. We show specific examples of parent-metabolite mimics with potent effects in vitro and in vivo. Furthermore, we show examples of emerging microbial ligand-receptor interactions and provide a context in which these ligands could be improved as potential drugs. A balanced conceptual advance is provided in which we also acknowledge potential pitfalls-hyperstimulation of finely balanced receptor-ligand interactions could also be detrimental. However, with balance, we provide examples of where this emerging concept needs to be tested.

\section{SIGNIFICANCE STATEMENT}

Microbial metabolite mimicry is a novel way to expand on the chemical repertoire of future drugs. The emerging concept is now explained using specific examples of the discovery of therapeutic leads from microbial metabolites.
This study was supported in part by The Peer Reviewed Medical Research Program - Investigator Initiated Research Award [Award No. W81XWH-17-10479], by National Institutes of Health (NIH) National Institute of Environmental Health Sciences [Grant R01 ES030197] and National Cancer Institute [Grant R01 CA222469], and The Czech Science Foundation [Grant 20-00449S].

We (S.M., Z.D.) have filed a patent application US 2019/0367475 A1: PXR agonists and uses thereof for gut barrier dysfunction and treatment prevention.

${ }^{1}$ Z.D. and M.K. equal contribution.

https://doi.org/10.1124/molpharm.120.000035.

\section{Biomimicry as an Innovation Concept}

Biomimicry is derived from the words, bios (Greek), or life or nature, and mimesis (Greek), or imitation (http://static.biomimicry. org/wp-content/uploads/2012/02/Biomimicry_Resource Handbook_Excerpt.pdf; Hargroves and Smith, 2006). Various industries have adopted biomimicry-based approaches for innovative solutions (https://www.weforum.org/agenda/2018/ 03/biomimicry-a-new-approach-to-innovation-inspired-bynature; Hu et al., 2019; Wood, 2019; Falanga et al., 2020). In medicine, biomimicry involves developing analogs of host

ABBREVIATIONS: AhR, aryl hydrocarbon receptor; DAT, desaminotyrosine; FXR, farnesol X receptor; GPCR (GPR), G-protein-coupled receptor; IA, indoleacrylate; IDO1, indoleamine 2,3-dioxygenase 1; Nrf2, nuclear factor erythroid 2-related factor 2; OCA, obeticholic acid; PXR, pregnane X receptor; SCFA, short-chain fatty acid; UroA, urolithin $A$. 
endogenous molecules that have evolutionarily adapted to target a given receptor and induce a favorable outcome. For instance, the soil bacterium Sorangium cellulosum (Gerth et al., 1994; Molnar et al., 2000) has likely evolved in its capacity for epothilone biosynthesis to ensure optimal fitness in its environment (Tang et al., 2000; Gerth et al., 2003; Rachid et al., 2007; Altmann et al., 2009). Epothilones have diverse therapeutic activity in host organisms as anti-inflammatory agents and antibiotics as well as antiviral, anticancer, and other types of therapies (Gerth et al., 1996). Modifying the oxidation states of a parental epothilone compound could give rise to a class of epothilone analogs with improved cytotoxic potential for cancer therapy (Tang et al., 2003). Indeed, a synthetic analog of microbial epothilones, Bristol Myers Squibb 247550 (ixabepilone), targets microtubules in mammalian cells and is approved for the treatment of breast cancer (Li et al., 2017). Beyond cancer indications, obeticholic acid (OCA), or Ocaliva, an analog of the endogenous farnesol X receptor (FXR) ligand chenodeoxycholic acid, has exhibited clinical benefit for primary biliary cholangitis (Pellicciari et al., 2002; Markham and Keam, 2016). Much effort has been allocated toward deriving therapeutics from potent metabolites produced by soil and marine bacteria. Here we will make the case that isolation of weak metabolites from the human commensal microbiome may increase the library of candidate metabolite analogs with therapeutic potential.

Recent evidence indicates that the human microbiome of a $70-\mathrm{kg}$ reference male contains approximately $3.8 \times 10^{13}$ cells, which is roughly equivalent to the number of human cells in the body (Sender et al., 2016). Although these figures remain best estimates, the massive numbers of microbes present in our bodies underscore their ability to expand the metabolic capabilities of the human organism. For example, in the gut, there are over 3-9 million unique bacterial genes (Yang et al., 2009; Qin et al., 2010). We may speculate that bacterial metabolic pathways are equally as diverse, and that their chemical compounds are highly varied in structure and function. Some metabolites derived from the commensal human microbiome exhibit therapeutic properties (Dobson et al., 2009; Saha et al., 2016; Descamps et al., 2019; Skelly et al., 2019). Metabolites with known therapeutic potential are likely to be only a fraction of the total therapeutic repertoire of microbial metabolites, as suggested in metabolomic studies (Folberth et al., 2020).

\section{Microbial Metabolite Mimicry as a Drug Discovery Concept}

We recently described microbial metabolite mimicry as an emerging concept for drug discovery (Dvořák et al., 2020). Based on the potential for improving the potency, selectivity, or pharmacokinetics of weak to moderate natural ligands, microbial metabolite mimics may simultaneously minimize off-target liabilities as a means to expand chemical and drug repertoires (Fig. 1). The feasibility of this approach has been demonstrated through the recent preclinical development of several mimics with promising results.

\section{Examples of Microbial Metabolite Mimicry in Drug Discovery}

Indole Mimics and Inflammation. Microbial metabolism of dietary L-tryptophan yields a number of indole-containing

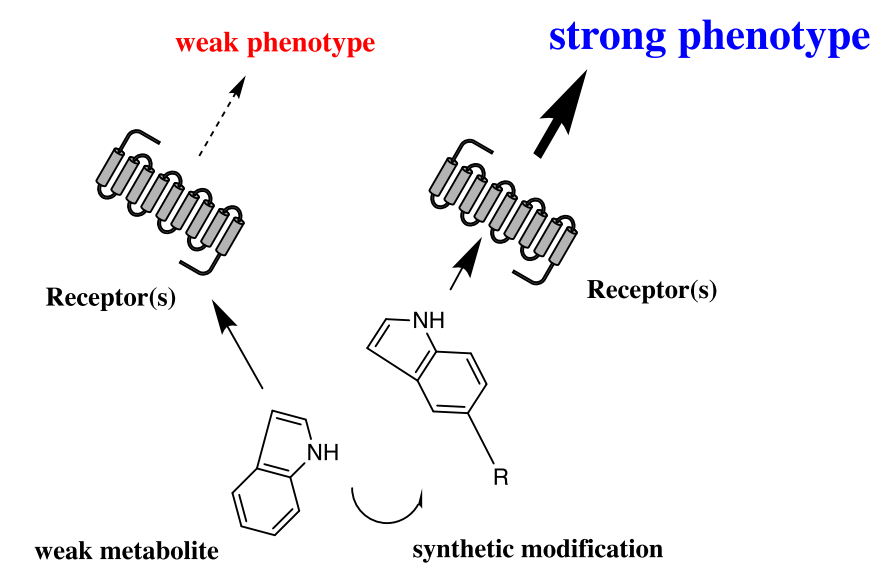

(n)

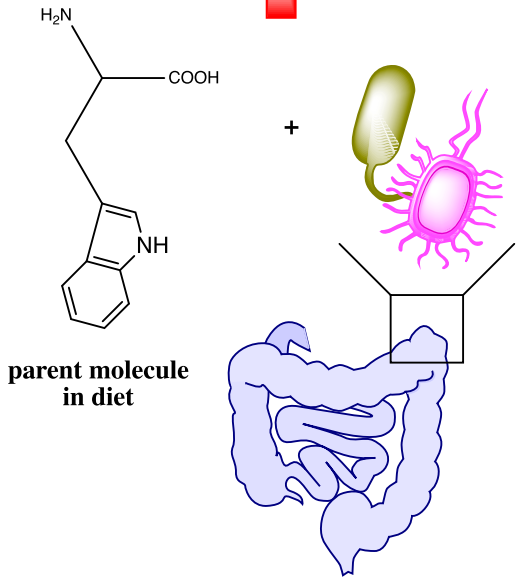

Fig. 1. Conceptual schematic of microbial metabolite mimicry. The intestine (blue) harbors many bacteria (multicolored). Some of these bacteria produce metabolites from a parent molecule in the diet (e.g., L-tryptophan) with weak receptor modulation, represented by an indole structure (weak metabolite). In applying a biomimicry-focused approach, modulating the chemical structure of a weak metabolite (synthetic modification) may produce a more potent modulator of a given receptor, thereby altering host phenotypes. These biomimics are chemically similar to the parent metabolite, and their off-target liabilities are likely to be less than that of xenobiotic structures, as demonstrated for indole mimics (Venkatesh et al., 2014).

metabolites (Roager and Licht, 2018) that are present and biologically active in rodents and humans (Vyhlídalová et al., 2020). These metabolites are diverse, and many have effects on multiple host receptors (Kim, 2018). For instance, the combination of indole (traditionally an aryl hydrocarbon receptor ligand) and its metabolite indole propionic acid was shown to activate the human pregnane $\mathrm{X}$ receptor (PXR). PXR agonism then regulates intestinal inflammation via the toll-like receptor 4-nuclear factor- $\kappa$ B pathway (Venkatesh et al., 2014). Subsequently, the same group developed indole-indole propionic acid pharmacophore analogs like Felix Kopp Kortagere 6 that exhibited potent PXR-dependent anti-inflammatory activity in mice (Nuzzo and Brown, 2020).

Indole can act upon other receptors important to hosting physiology, including the aryl hydrocarbon receptor (AhR). Activation of AhR by indole or other tryptophan metabolites has been shown to protect against colitis in mice (Rogala et al., 2020). There are several microbial-derived tryptophan metabolites that act as AhR agonists and can modulate the gastrointestinal 
immune cells, enhance barrier function, and inhibit intestinal inflammation (Zelante et al., 2013; Rothhammer et al., 2016; Aoki et al., 2018; Natividad et al., 2018; Roager and Licht, 2018; Vyhlidalova et al., 2020). In this context, rationally designed indole analogs have been shown to act as potent AhR ligands that abrogate intestinal inflammation (Kawai et al., 2017; Chen et al., 2020). Since these are derived from parent molecules for which the host is likely tolerized, their toxicity is likely to be less than xenobiotics (Chen et al., 2020; Dvoŕák et al., 2020). More recently, $\mathrm{N}$-acetylserotonin, a tryptophan metabolite produced along the serotonin pathway, acts as a positive allosteric modulator of indoleamine 2,3-dioxygenase 1 (IDO1) (Sonowal et al., 2017). IDO1 is an immunoregulatory enzyme involved in converting tryptophan to kynurenine, an endogenous AhR agonist (Mondanelli et al., 2020). The allosteric agonism of IDO1 by $N$-acetylserotonin protected mice from neuroinflammation and restored physiologic IDO1 activity in peripheral blood mononuclear cells from patients with relapsing-remitting multiple sclerosis (Mondanelli et al., 2020). Stable analogs of kynurenine may represent a feasible modality for AhR-targeted anti-inflammatory drug indications for autoimmunity. New classes of microbial-derived AhR ligands have been described (e.g., 1,4-dihydroxy-2-naphthoic acid) (Fukumoto et al., 2014). 1,4Dihydroxy-2-naphthoic acid inhibits dextran sulfate sodium colitis in mice, and these ligands would appear to be a scaffold for a new class of AhR-active drugs (Fukumoto et al., 2014).

Apart from autoimmunity, pathogens also exploit the role of indole metabolism in maintaining homeostasis in the host. The pathobiont Klebsiella oxytoca secretes the indole analog tilivalline to induce antibiotic-associated hemorrhagic colitis (Stampfer et al., 2017). Substantially simplified tilivalline mimics block the production of tilivalline (von Tesmar et al., 2018). As these molecules mimic endogenous indoles produced by gut bacteria, they are likely to be nontoxic and improve health span (Sonowal et al., 2017).

Microbial Short-Chain Fatty Acids and Host G-Protein-Coupled Receptors in Health. Short-chain fatty acids (SCFAs) are produced by intestinal bacterial fermentation of nonabsorbed dietary carbohydrates. They have profound and generally homeostatic effects in the intestine (Kaiko et al., 2016; Chen et al., 2019). There are several SCFA receptors (e.g., GPR43, FFA2, FFA3, GPR109a) involved in the process of regulating colonic epithelial physiology and mucosal immune responses (Smith et al., 2013; Priyadarshini et al., 2018; Bolognini et al., 2019). SCFAs bind to their canonical receptors with weak affinity (micromolar range) (Husted et al., 2017). Homology and crystal structure models, along with mutagenesis and structure-function studies, have paved a clear road for the discovery of potent small-molecule SCFA analogs (Tikhonova, 2017). Alternatively, glycoengineering SCFAs onto a drug candidate improved pharmacologic properties with therapeutic potential for glycan-mediated pathologies (Saeui et al., 2018). SCFA-induced adipocyte differentiation can be enhanced using SCFA analogs (Jiang et al., 2013). SCFAs also exhibit weak inhibition of histone deacetylases. Specific zincchelating and motif-tethered SCFA analogs display nanomolar potency as histone deacetylase inhibitors (Lu et al., 2004), which suggests a feasible alternative source of chromatin-modifying drugs. Tributyrin, a prodrug of the SCFA butyrate, has more favorable pharmacokinetic properties than butyrate itself (Egorin et al., 1999) and, furthermore, was shown to have protective effects in ethanol-induced mouse models of intestinal barrier dysfunction and liver injury (Cresci et al., 2014, 2017). Similarly, tributyrin can decrease the fitness advantage of pathogens like Salmonella that depend on lactate metabolism (Gillis et al., 2018). SCFAs may be important as a novel type of "co-drug" that enhances AhR-mediated effects in the gut (Korecka et al., 2016; Jin et al., 2017). Moreover, there is evidence that some high-fiber diets can be metabolized to enhance SCFA production, and these can be used in human studies (Baxter et al., 2019).

Dietary Nutrients, Microbial Metabolites, and Infection. Desaminotyrosine (DAT) is a microbial degradation product of polyphenolic flavonoids (Lambert and Moss, 1980; Schoefer et al., 2003). Recent data suggest that DAT protects against influenza virus infection by inducing type I interferons (Steed et al., 2017). Diets rich in polyphenols could exert a positive influence on DAT production. Identification of the exact molecular targets of DAT is a vital first step toward realizing DAT mimics with improved potency. Although the deployment of better intestinal models of humans is needed for the evaluation of polyphenol bacterial metabolites on preclinical models (van Duynhoven et al., 2011), a few studies have emerged that associate polyphenol metabolites with host health, including one implicating the metabolism of phenolic acids in blueberries (Russell et al., 2007). In these models, defining the targets of phenolic acids would be essential prior to embarking on drug discovery through microbial metabolite mimicry.

Microbial Enzyme Inhibitors, Diabetes, and Obesity. As a proof of concept, acarbose ( $\alpha$-glucosidase inhibitor) is frequently used in diabetic patients to control glycemia and postload insulin levels (van de Laar et al., 2005). Acarbose is made by Actinoplanes sp. SE 50/110. It is a pseudotetrasaccharide and contains an aminocyclitol moiety, valienamine, which inactivates intestinal $\alpha$-glucosidase and sucrase. This combined effect decreases intestinal starch hydrolysis. Similarly, a host of inhibitors are derived from marine microbes, most with weak enzyme-inhibitor properties but with core pharmacophores that could be chemically modified (e.g., indole) (Gomez-Betancur et al., 2019). Amylase inhibitors are also positioned as drugs for weight loss (Mahmood, 2016). Despite the use of such inhibitors in diabetes (Jayaraj et al., 2013), long-term use warrants safe agents. To expand the drug repertoire by improving weak inhibitors, such as those obtained from microbial products (metabolites), mimicry would be applicable. For example, a few microbial $\alpha$-amylase inhibitors include paim (obtained from culture filtrates of Streptomyces corchorushii) and Taisho amylase inhibitor-A and Taisho amylase inhibitor-B (oligosaccharide compounds from Streptomyces calvus TM-521) (Demain and Sanchez, 2009). Lipstatin, a pancreatic lipase inhibitor produced by Streptomyces toxytricini, is used to treat obesity and diabetes (Híreš et al., 2018). A stable analog of lipstatin, tetrahydrolipstatin (orlistat), is Food and Drug Administration-approved for the treatment of obesity (Filippatos et al., 2008). The side effects of orlistat are minimal and manageable but do include nausea, vomiting, and diarrhea (Khalil et al., 2020). In rats, orlistat may increase aberrant crypt formation during chemically induced inflammation (Garcia et al., 2006).

\section{Emerging Opportunities}

Microbial Metabolites and G-Protein-Coupled Receptor Families. In addition to SCFAs, other human microbiome-specific metabolites (e.g., phenylpropanoic acid, cadaverine, 9,10-methylenehexadecanoic acid, and 
12-methyltetradecanoic acid) modulate host G-protein-coupled receptors (GPCRs) (Offermanns, 2017; Colosimo et al., 2019). The authors screened multiple fermentation fractions of bacterial culture broths from a simplified human microbiota simplified human microbiota consortium (Eun et al., 2014) and, after reverse-phase chromatography, applied the fractions to a multiwell 241 GPCR-specific engineered cell-line screen ( $\beta$-arrestin recruitment screen). Although some metabolites (e.g., nicotinic acid $\mathrm{EC}_{50} \sim 2.2 \mu \mathrm{M}$ for GPR109A) were inherently potent activators of GPCRs, there were several new metabolites with weak agonist activity (e.g., 3-hydroxyoctanoic acid $\mathrm{EC}_{50} \sim 304 \mu \mathrm{M}$; phenylpropanoic acid, a bacterial ligand, $\mathrm{EC}_{50} \sim 208 \mu \mathrm{M}$ for GPR109B). Homology models, based on prior knowledge of GPR109A (Tunaru et al., 2005), and mutagenesis studies could be leveraged to define the ligand-binding pocket interactions for GPR109B with weak ligands; this could be useful for deriving phenylpropanoic acid mimics. Similar examples are shown by Cohen et al. (2017), whereby a microbiome-synthetic gene-therapy approach is applied toward GPR119-targeted therapy, given that human microbiota produce N-acyl amides that mimic human GPR119 ligands. Furthermore, defining GPCRs with dominant roles for a given ligand would be important to assess so that the correct receptor or set of receptors can be chosen to perform a library screen of mimics (Bolognini et al., 2019).

Bile Acids, Microbial Metabolites, and FXR. The FXR is a major target for liver disease prevention (Hoofnagle, 2020), with the primary bile acid chenodeoxycholic acid as the endogenous ligand. FXR agonists now in clinical use include OCA (Pellicciari et al., 2002, 2018) and cilofexor (a non-bile acid synthetic FXR agonist), which have inspired drug development for a variety of hepatic diseases (Gioiello et al., 2014). The results of this study by Pellicciari and colleagues identified a key small pocket in the receptor ligand-binding domain that accommodates small hydrophobic groups at the C6 $\alpha$-position of chenodeoxycholic acid. In particular, the ethyl moiety perfectly fits the $10-\AA$ cavity, leading to a 100 -fold increase in FXR activity, and has become a key structural motif of a novel, potent bile acid-based modulator (findings made by Fraydoon Rastinejad). In 2016, OCA reached patients affected by primary biliary cholangitis and is currently being evaluated in patients with nonalcoholic steatohepatitis (Pellicciari et al., 2018). More recently, microbial amino-acid conjugations of the host bile acids, phenylalanocholic, tyrosocholic, and leucocholic acid, have been identified as FXR agonists (Quinn et al., 2020). Complementing these are other secondary microbial metabolites of bile acids like isodeoxycholate (Campbell et al., 2020). Using these templates, mimics could improve potency while limiting side effects (Quinn et al., 2020).

Dummy Analogs. The underlying rationale for the design of dummy analogs is to mimic a given bioactive molecule to promote competitive inhibition of a given target. Imidazole propionate, a human and rodent microbiome-specific metabolite, blunts insulin-induced mammalian target of rapamycin complex 1 activation, impairing glycolysis (Koh et al., 2018). The authors defined the alternative p38 pathway as a target of imidazole propionate. Thus, dummy imidazole analogs could blunt the effects of imidazole propionate on p38 via competitive binding. Importantly, the design of an imidazole analog library is reasonable given the simplicity of the biosynthesis of imidazole propionate from histidine (Koh et al., 2018).
Microbial Metabolites and Antioxidant Drug Discovery. Nuclear factor erythroid 2-related factor 2 (Nrf2) is a global regulator of the antioxidant response that is evolutionarily conserved. A resident mucin-degrading bacterium Peptostreptococcus russellii produces the tryptophan metabolite indoleacrylate (IA), which activates Nrf2 and improves barrier function (Wlodarska et al., 2017). Although it remains unclear whether IA directly binds Nrf2, deeper investigation could reveal methods to mimic IA. Other small-molecule scaffolds, such as oxo fatty acids that activate the Nrf2antioxidant responsive element pathway, can also be used to enhance the potency of mimics (Sofyana et al., 2020). Urolithin A (UroA) is a major microbial metabolite derived from polyphenolics of berries and pomegranate fruits. UroA displays anti-inflammatory and antioxidative activities yet weak potency. A recent report demonstrates the therapeutic potential of a more potent UroA analog UAS03 that enhanced barrier function and decreased inflammation in mice (Singh et al., 2019). It is to be noted that UroA could also act via the AhR pathway as a ligand, and therefore, its effects on any phenotype could be due to pleiotropic molecular mechanisms (Muku et al., 2018; Pernomian et al., 2020). In this context, vitamin metabolism by gut bacteria could affect the overall health of the host. For example, vitamin $\mathrm{E}$ acetate is metabolized by Lactobacillus acidophilus NCFM (Roager et al., 2014) similarly to the metabolism of natural forms of vitamin $\mathrm{E}, \alpha$-tocopherol, and $\gamma$-tocopherol by the intestinal microbiota of rats (Ran et al., 2019). Understanding the consequences of vitamin $\mathrm{E}$ metabolites and mimics on host health requires further study. These investigations should be coupled with defining not only host phenotypes but specific protein targets affected by those vitamins and their bioactive long-chain metabolites (e.g., garcinoic acid) (Bartolini et al., 2020). Along with PXR, these targets appear to include other nuclear receptors (e.g., peroxisome proliferator-activated receptor- $\gamma$ ) recently reviewed elsewhere (Torquato et al., 2020) and the anti-inflammatory protein 5-lipoxygenase (Pein et al., 2018). In another example, microbiome-derived ascorbate inhibits glucose transporter 1 in human $\mathrm{CD}_{4}^{+}$ effector T cells, inducing apoptosis (Chang et al., 2019). However, alternative mechanisms of ascorbate delivery to sites of local inflammation cannot be excluded (Wang et al., 1997). Ascorbate mimics that potently inhibit glucose transporter 1 , which is upregulated in activated T cells, could be a unique way to develop drugs targeting Crohn disease and other T cell-mediated nonmalignant disorders. In this regard, since multiple transporters are involved in ascorbate transport in different tissues, analogs specific to each transporter may also be feasible and could have reduced systemic, offtarget toxicity (Corpe et al., 2005).

Microbial Metabolites and Mimics as Alternatives to Antibiotics. Discovery of new antibiotics is of crucial importance to combat antimicrobial resistance, with a very significant impact on global health and the agriculture industry. Microbial metabolite mimicry can certainly be applied here, wherein most clinically used antibiotics are microbially derived (Lewis, 2020). In the pork industry, early weaning of piglets improves the reproductive cycle in sows (Campbell et al., 2013); however, early weaning often causes stressinduced diarrhea in the piglets that can respond to antibiotics (Lallès et al., 2007), although antibiotic resistance remains a major problem (Allen et al., 2014; Chen et al., 2018). More 
recently, it has been shown that fecal transplants from resistant to susceptible early-weaned piglets reduce this stress-induced diarrhea ( $\mathrm{Hu}$ et al., 2018). Two dominant strains, Lactobacillus gasseri LA39 and Lactobacillus frumenti, were identified from diarrhea-resistant feces that also confer resistance to diarrhea in susceptible piglets. The principal inhibitor that prevents diarrhea is the bacterial circular peptide gassericin $\mathrm{A}$, which mediates its effect via host keratin 19 (Hu et al., 2018). It remains unclear whether this peptide fragment is optimized for keratin 19 binding, and optimizing the peptide sequence for this effect could present new therapeutic modalities.

Microbial Metabolites and Mimics to Shape Microbial Diversity. Some microbial metabolites can shape the emergence or loss of microbial diversity (Lilja and Johnson, 2017; Goldschmidt et al., 2018; Douglas, 2020). Microbial metabolite mimics with specific antibacterial effects could be screened against a consortium of intestinal bacteria in in vitro- and in consortia-inoculated germ-free mice to look for diversity control as a means to aide in host disease control. In this way, mimicry allows for expanding the metabolite repertoire to diversify the microbiome and maintain homeostasis of host health (Haag and Siegmund, 2014). This might be particularly relevant to the rapidly increasing Westernpredominant inflammatory conditions associated with dysbiosis, such as inflammatory bowel diseases (Crohn disease and ulcerative colitis), metabolic syndrome, obesity, fatty liver disease, and inflammatory arthritis. In this respect, a dietary approach could be taken. A natural flavanol Kaempferol demonstrated significant activity against collagen-induced arthritis in mice when administered orally but not intraperitoneally, and Kaempferol was retained in the gut and diversified the microbiota; these data support the contribution of microbiome diversity toward the therapeutic effect (Aa et al., 2020). Kaempferol mimics with potent microbial reshaping ability could be designed for the treatment of arthritis (Aa et al., 2020).

The examples provided in this section of emerging opportunities demonstrate specific pathways in which microbial metabolites produced by bacteria in the host effectively engage one or more receptors in tissues. There is a clear phenotype observed when the metabolite engages the host tissue receptor. Indeed, modifying the metabolite to either improve its potency or to make the metabolite more stable could provide broad approaches toward improving drug discovery.

\section{Future Efforts}

Based on the rationale and examples presented, we recommend exploiting metabolically focused host-microbe relationships for future drug discovery using the microbial metabolite mimicry approach. These efforts should be coupled with medicinal chemistry and medium to high-throughput host phenotype assays. In some cases, multispecific small molecules may be feasible, especially when targeting a protein complex, as has been shown for antibodies (Deshaies, 2020). The chemistry should be simple, possibly automated, and integrated in innovative discovery platforms (Gioiello et al., 2020) to keep the process more efficient and at a low cost. Emphasis should also be on utilizing microbial chemistry pathways and well-designed and well-engineered biocatalysts to synthesize novel mimics-perhaps using concepts of directed evolution of salient genes involved in the metabolite-synthesis pathway (Chen and Arnold, 1993).

In summary, several microbial metabolites have weak interactions with host receptors and offer the potential to generate mimics with increased binding affinities that will produce little to no toxicity when applied as therapies due to the host's tolerance to the native forms of these metabolites. Microbe-host interactions are critical to host physiology, and these relationships could be exploited chemically to drive favorable interactions. In drug discovery, in comparison with efforts on soil and marine bacterial metabolites, the human microbiome offers many such interactions that warrant chemical mimicry. Although these mimics could suffer the same metabolic fate as other xenobiotics, by keeping the chemistry simple and with a full understanding of its metabolic fate/ liabilities, it is possible to design better drugs.

Evolutionarily, it is also possible that these weaker receptor-ligand interactions are advantageous to the host to prevent hyperstimulation of the respective receptors, which may in fact have detrimental consequences. Furthermore, not all microbial metabolites benefit the host because some may drive inflammation and carcinogenesis (Nyangale et al., 2012; Windey et al., 2012; O'Keefe, 2016). As such, in developing this new field of pharmacology, it is essential to understand the balance between binding affinities and physiologic outcomes to fine-tune receptor-ligand interactions for optimal health outcomes.

\section{Acknowledgments}

The authors thank Susan Horwitz (Albert Einstein College of Medicine, Bronx, NY) and Rolf Muller (Saarland University, Saarbrücken, Germany) for insightful and helpful discussions.

\section{Authorship Contributions}

Participated in research design: Dvorak, Klapholz, Gioiello, Pelliciari, Galli, March, Sartor, Levy, Mani.

Wrote or contributed to the writing of the manuscript: Dvorak, Klapholz, Burris, Willing, Gioiello, Pelliciari, Galli, March, Sartor, O’Keefe, Kim, Levy, Mani.

\section{References}

Aa LX, Fei F, Qi Q, Sun RB, Gu SH, Di ZZ, Aa JY, Wang GJ, and Liu CX (2020) Rebalancing of the gut flora and microbial metabolism is responsible for the antiarthritis effect of kaempferol. Acta Pharmacol Sin 41:73-81.

Allen HK, Trachsel J, Looft T, and Casey TA (2014) Finding alternatives to antibiotics. Ann N Y Acad Sci 1323:91-100.

Altmann KH, Kinghorn AD, Mulzer JH, Höfle G, Müller R, and Prantz K (2009) The Epothilones: An Outstanding Family of Anti-Tumor Agents: From Soil to the Clinic, Springer, Vienna, Austria.

Aoki R, Aoki-Yoshida A, Suzuki C, and Takayama Y (2018) Indole-3-pyruvic acid, an aryl hydrocarbon receptor activator, suppresses experimental colitis in mice. $J$ Immunol 201:3683-3693.

Bartolini D, De Franco F, Torquato P, Marinelli R, Cerra B, Ronchetti R, Schon A, Fallarino F, De Luca A, Bellezza G, et al. (2020) Garcinoic acid is a natural and selective agonist of pregnane X receptor. J Med Chem 63:3701-3712.

Baxter NT, Schmidt AW, Venkataraman A, Kim KS, Waldron C, and Schmidt TM (2019) Dynamics of human gut microbiota and short-chain fatty acids in response to dietary interventions with three fermentable fibers. MBio 10:e02566-18.

Bolognini D, Barki N, Butcher AJ, Hudson BD, Sergeev E, Molloy C, Moss CE, Bradley SJ, Le Gouill C, Bouvier M, et al. (2019) Chemogenetics defines receptormediated functions of short chain free fatty acids. Nat Chem Biol 15:489-498.

Campbell C, McKenney PT, Konstantinovsky D, Isaeva OI, Schizas M, Verter J, Mai C, Jin W-B, Guo C-J, Violante S, et al. (2020) Bacterial metabolism of bile acids promotes generation of peripheral regulatory T cells. Nature 581:475-479.

Campbell JM, Crenshaw JD, and Polo J (2013) The biological stress of early weaned piglets. J Anim Sci Biotechnol 4:19.

Chang Y-L, Rossetti M, Vlamakis H, Casero D, Sunga G, Harre N, Miller S, Humphries R, Stappenbeck T, Simpson KW, et al. (2019) A screen of Crohn's disease-associated microbial metabolites identifies ascorbate as a novel metabolic inhibitor of activated human T cells. Mucosal Immunol 12:457-467.

Chen J, Haller CA, Jernigan FE, Koerner SK, Wong DJ, Wang Y, Cheong JE, Kosaraju R, Kwan J, Park DD, et al. (2020) Modulation of lymphocyte-mediated 
tissue repair by rational design of heterocyclic aryl hydrocarbon receptor agonists. Sci Adv 6:eaay8230.

Chen J, Zhao K-N, and Vitetta L (2019) Effects of intestinal microbial ${ }^{-}$elaborated butyrate on oncogenic signaling pathways. Nutrients 11:1026.

Chen K and Arnold FH (1993) Tuning the activity of an enzyme for unusual environments: sequential random mutagenesis of subtilisin $\mathrm{E}$ for catalysis in dimethylformamide. Proc Natl Acad Sci USA 90:5618-5622.

Chen Z, Wang Z, Ren J, and Qu X (2018) Enzyme mimicry for combating bacteria and biofilms. Acc Chem Res 51:789-799.

Cohen LJ, Esterhazy D, Kim S-H, Lemetre C, Aguilar RR, Gordon EA, Pickard AJ, Cross JR, Emiliano AB, Han SM, et al. (2017) Commensal bacteria make GPCR ligands that mimic human signalling molecules. Nature 549:48-53.

Colosimo DA, Kohn JA, Luo PM, Piscotta FJ, Han SM, Pickard AJ, Rao A, Cross JR, Cohen LJ, and Brady SF (2019) Mapping interactions of microbial metabolites with human G-protein-coupled receptors. Cell Host Microbe 26:273-282.e7.

Corpe CP, Lee J-H, Kwon O, Eck P, Narayanan J, Kirk KL, and Levine M (2005) 6 Bromo-6-deoxy-L-ascorbic acid: an ascorbate analog specific for Na+-dependent vitamin $\mathrm{C}$ transporter but not glucose transporter pathways. J Biol Chem 280: $5211-5220$

Cresci GA, Bush K, and Nagy LE (2014) Tributyrin supplementation protects mice from acute ethanol-induced gut injury. Alcohol Clin Exp Res 38:1489-1501.

Cresci GA, Glueck B, McMullen MR, Xin W, Allende D, and Nagy LE (2017) Prophylactic tributyrin treatment mitigates chronic-binge ethanol-induced intestinal barrier and liver injury. J Gastroenterol Hepatol 32:1587-1597.

Demain AL and Sanchez S (2009) Microbial drug discovery: 80 years of progress. $J$ Antibiot (Tokyo) 62:5-16.

Descamps HC, Herrmann B, Wiredu D, and Thaiss CA (2019) The path toward using microbial metabolites as therapies. EBioMedicine 44:747-754.

Deshaies RJ (2020) Multispecific drugs herald a new era of biopharmaceutical innovation. Nature 580:329-338.

Dobson PD, Patel Y, and Kell DB (2009) 'Metabolite-likeness' as a criterion in the design and selection of pharmaceutical drug libraries. Drug Discov Today 14 $31-40$

Douglas AE (2020) The microbial exometabolome: ecological resource and architect of microbial communities. Philos Trans R Soc Lond B Biol Sci 375:20190250.

Dvořák Z, Kopp F, Costello CM, Kemp JS, Li H, Vrzalová A, Stěpánková M, Bartoňková I, Jiskrová E, Poulíková K, et al. (2020) Targeting the pregnane X receptor using microbial metabolite mimicry. EMBO Mol Med 12:e11621.

Egorin MJ, Yuan ZM, Sentz DL, Plaisance K, and Eiseman JL (1999) Plasma pharmacokinetics of butyrate after intravenous administration of sodium butyrate or oral administration of tributyrin or sodium butyrate to mice and rats. Cancer Chemother Pharmacol 43:445-453.

Eun CS, Mishima Y, Wohlgemuth S, Liu B, Bower M, Carroll IM, and Sartor RB (2014) Induction of bacterial antigen-specific colitis by a simplified human microbiota consortium in gnotobiotic interleukin-10-/- mice. Infect Immun 82:2239-2246.

Falanga D, Kleber K, and Scaramuzza D (2020) Dynamic obstacle avoidance for quadrotors with event cameras. Sci Robot 5:eaaz9712.

Filippatos TD, Derdemezis CS, Gazi IF, Nakou ES, Mikhailidis DP, and Elisaf MS (2008) Orlistat-associated adverse effects and drug interactions: a critical review. Drug Saf 31:53-65.

Folberth J, Begemann K, Jöhren O, Schwaninger M, and Othman A (2020) MS ${ }^{2}$ and LC libraries for untargeted metabolomics: enhancing method development and identification confidence. J Chromatogr B Analyt Technol Biomed Life Sci 1145 122105.

Fukumoto S, Toshimitsu T, Matsuoka S, Maruyama A, Oh-Oka K, Takamura T, Nakamura Y, Ishimaru K, Fujii-Kuriyama Y, Ikegami S, et al. (2014) Identification of a probiotic bacteria-derived activator of the aryl hydrocarbon receptor that inhibits colitis. Immunol Cell Biol 92:460-465.

Garcia SB, Barros LT, Turatti A, Martinello F, Modiano P, Ribeiro-Silva A, Vespúcio MV, and Uyemura SA (2006) The anti-obesity agent Orlistat is associated to increase in colonic preneoplastic markers in rats treated with a chemical carcinogen. Cancer Lett 240:221-224.

Gerth K, Bedorf N, Höfle G, Irschik H, and Reichenbach H (1996) Epothilons A and $\mathrm{B}$ : antifungal and cytotoxic compounds from Sorangium cellulosum (Myxobacteria). Production, physico-chemical and biological properties. J Antibiot (Tokyo) 49 $560-563$

Gerth K, Bedorf N, Irschik H, Höfle G, and Reichenbach H (1994) The soraphens: a family of novel antifungal compounds from Sorangium cellulosum (Myxobacteria). I. Soraphen A1 alpha: fermentation, isolation, biological properties. $J$ Antibiot (Tokyo) 47:23-31.

Gerth K, Pradella S, Perlova O, Beyer S, and Müller R (2003) Myxobacteria: proficient producers of novel natural products with various biological activities--past and future biotechnological aspects with the focus on the genus Sorangium. $J$ Biotechnol 106:233-253.

Gillis CC, Hughes ER, Spiga L, Winter MG, Zhu W, Furtado de Carvalho T, Chanin RB, Behrendt CL, Hooper LV, Santos RL, et al. (2018) Dysbiosis-associated change in host metabolism generates lactate to support Salmonella growth. Cell Host Microbe 23:54-64.e6.

Gioiello A, Cerra B, Mostarda S, Guercini C, Pellicciari R, and Macchiarulo A (2014) Bile acid derivatives as ligands of the farnesoid $\mathrm{x}$ receptor: molecular determinants for bile acid binding and receptor modulation. Curr Top Med Chem 14:2159-2174.

Gioiello A, Piccinno A, Lozza AM, and Cerra B (2020) The medicinal chemistry in the era of machines and automation: recent advances in continuous flow technology. Med Chem 63:6624-6647.

Goldschmidt F, Regoes RR, and Johnson DR (2018) Metabolite toxicity slows local diversity loss during expansion of a microbial cross-feeding community. ISME J 12 136-144.

Gómez-Betancur I, Zhao J, Tan L, Chen C, Yu G, Rey-Suárez P, and Preciado L (2019) Bioactive compounds isolated from marine bacterium Vibrio neocaledonicus and their enzyme inhibitory activities. Mar Drugs 17:401.
Haag LM and Siegmund B (2014) Exploring \& exploiting our 'other self' - does the microbiota hold the key to the future therapy in Crohn's? Best Pract Res Clin Gastroenterol 28:399-409.

Hargroves K and Smith M (2006) Innovation inspired by nature: Biomimicry. Ecos 129:27-29.

Híreš M, Rapavá N, Šimkovič M, Varečka Ľ, Berkeš D, and Kryštofová S (2018) Development and optimization of a high-throughput screening assay for rapid evaluation of lipstatin production by Streptomyces strains. Curr Microbiol 75:580-587.

Hoofnagle JH (2020) FXR agonists as therapy for liver disease. Hepatology 72:1-3.

Hu C, Chen Y, Tan MJA, Ren K, and Wu H (2019) Microfluidic technologies for vasculature biomimicry. Analyst (Lond) 144:4461-4471.

Hu J, Ma L, Nie Y, Chen J, Zheng W, Wang X, Xie C, Zheng Z, Wang Z, Yang T, et al (2018) A microbiota-derived bacteriocin targets the host to confer diarrhea resistance in early-weaned piglets. Cell Host Microbe 24:817-832.e8.

Husted AS, Trauelsen M, Rudenko O, Hjorth SA, and Schwartz TW (2017) GPCRmediated signaling of metabolites. Cell Metab 25:777-796.

Jayaraj S, Suresh S, and Kadeppagari R-K (2013) Amylase inhibitors and their biomedical applications. Starke 65:535-542.

Jiang W, Sunkara LT, Zeng X, Deng Z, Myers SM, and Zhang G (2013) Differential regulation of human cathelicidin LL-37 by free fatty acids and their analogs. Peptides 50:129-138.

Jin UH, Cheng Y, Park H, Davidson LA, Callaway ES, Chapkin RS, Jayaraman A, Asante A, Allred C, Weaver EA, et al. (2017) Short chain fatty acids enhance aryl hydrocarbon $(\mathrm{Ah})$ responsiveness in mouse colonocytes and caco-2 human colon cancer cells. Sci Rep 7:10163.

Kaiko GE, Ryu SH, Koues OI, Collins PL, Solnica-Krezel L, Pearce EJ, Pearce EL, Oltz EM, and Stappenbeck TS (2016) The colonic crypt protects stem cells from microbiota-derived metabolites. Cell 165:1708-1720.

Kawai S, Iijima H, Shinzaki S, Hiyama S, Yamaguchi T, Araki M, Iwatani S, Shiraishi E, Mukai A, Inoue T, et al. (2017) Indigo Naturalis ameliorates murine dextran sodium sulfate-induced colitis via aryl hydrocarbon receptor activation. $J$ Gastroenterol 52:904-919.

Khalil H, Ellwood L, Lord H, and Fernandez R (2020) Pharmacological treatment for obesity in adults: an umbrella review. Ann Pharmacother 54:691-705.

Kim CH (2018) Immune regulation by microbiome metabolites. Immunology 154: 220-229.

Koh A, Molinaro A, Ståhlman M, Khan MT, Schmidt C, Mannerås-Holm L, Wu H, Carreras A, Jeong H, Olofsson LE, et al. (2018) Microbially produced imidazole propionate impairs insulin signaling through mTORC1. Cell 175:947-961.e17.

Korecka A, Dona A, Lahiri S, Tett AJ, Al-Asmakh M, Braniste V, D'Arienzo R, Abbaspour A, Reichardt N, Fujii-Kuriyama Y, et al. (2016) Bidirectional communication between the Aryl hydrocarbon Receptor (AhR) and the microbiome tunes host metabolism. NPJ Biofilms Microbiomes 2:16014.

Lallès J-P, Bosi P, Smidt H, and Stokes CR (2007) Nutritional management of gut health in pigs around weaning. Proc Nutr Soc 66:260-268.

Lambert MA and Moss CW (1980) Production of p-hydroxyhydrocinnamic acid from tyrosine by Peptostreptococcus anaerobius. J Clin Microbiol 12:291-293.

Lewis K (2020) The science of antibiotic discovery. Cell 181:29-45.

Li J, Ren J, and Sun W (2017) Systematic review of ixabepilone for treating metastatic breast cancer. Breast Cancer 24:171-179.

Lilja EE and Johnson DR (2017) Metabolite toxicity determines the pace of molecular evolution within microbial populations. BMC Evol Biol 17:52.

Lu Q, Yang YT, Chen CS, Davis M, Byrd JC, Etherton MR, Umar A, and Chen CS (2004) Zn2+-chelating motif-tethered short-chain fatty acids as a novel class of histone deacetylase inhibitors. J Med Chem 47:467-474.

Mahmood N (2016) A review of $\alpha$-amylase inhibitors on weight loss and glycemic control in pathological state such as obesity and diabetes. Comp Clin Pathol 25: $1253-1264$

Markham A and Keam SJ (2016) Obeticholic acid: first global approval. Drugs 76 $1221-1226$

Molnár I, Schupp T, Ono M, Zirkle R, Milnamow M, Nowak-Thompson B, Engel N, Toupet C, Stratmann A, Cyr DD, et al. (2000) The biosynthetic gene cluster for the microtubule-stabilizing agents epothilones A and B from Sorangium cellulosum So ce90. Chem Biol 7:97-109.

Mondanelli G, Coletti A, Greco FA, Pallotta MT, Orabona C, Iacono A, Belladonna ML, Albini E, Panfili E, Fallarino F, et al. (2020) Positive allosteric modulation of indoleamine 2,3-dioxygenase 1 restrains neuroinflammation. Proc Natl Acad Sci USA 117:3848-3857.

Muku GE, Murray IA, Espín JC, and Perdew GH (2018) Urolithin A is a dietary microbiota-derived human aryl hydrocarbon receptor antagonist. Metabolites 8:86.

Natividad JM, Agus A, Planchais J, Lamas B, Jarry AC, Martin R, Michel ML, Chong-Nguyen C, Roussel R, Straube M, et al. (2018) Impaired aryl hydrocarbon receptor ligand production by the gut microbiota is a key factor in metabolic syndrome. Cell Metab 28:737-749.e4.

Nuzzo A and Brown JR (2020) Microbiome metabolite mimics accelerate drug discovery. Trends Mol Med 26:435-437.

Nyangale EP, Mottram DS, and Gibson GR (2012) Gut microbial activity, implications for health and disease: the potential role of metabolite analysis. J Proteome Res 11:5573-5585.

Offermanns S (2017) Hydroxy-carboxylic acid receptor actions in metabolism. Trends Endocrinol Metab 28:227-236.

O'Keefe SJ (2016) Diet, microorganisms and their metabolites, and colon cancer. Nat Rev Gastroenterol Hepatol 13:691-706.

Pein H, Ville A, Pace S, Temml V, Garscha U, Raasch M, Alsabil K, Viault G, Dinh CP, Guilet D, et al. (2018) Endogenous metabolites of vitamin E limit inflammation by targeting 5-lipoxygenase. Nat Commun 9:3834.

Pellicciari R, Fiorucci S, Camaioni E, Clerici C, Costantino G, Maloney PR, Morelli A Parks DJ, and Willson TM (2002) 6 $\alpha$-ethyl-chenodeoxycholic acid (6-ECDCA), a potent and selective FXR agonist endowed with anticholestatic activity. J Med Chem 45 3569-3572. 
Pellicciari R, Pruzanski M, and Gioiello A (2018) The Discovery of Obeticholic Acid (Ocaliva $^{\mathrm{TM}}$ ): First-in-Class FXR Agonist, in Successful Drug Discovery, Volume 3 (Fischer J, Klein C, and Childers W eds) pp 197-244, Wiley-VCH Verlag GmbH \& Co. KGaA, Weinheim.

Pernomian L, Duarte-Silva M, and de Barros Cardoso CR (2020) The Aryl Hydrocarbon Receptor (AHR) as a potential target for the control of intestinal inflammation: insights from an immune and bacteria sensor receptor. Clin Rev Allergy Immunol DOI: 10.1007/s12016-020-08789-3 [published ahead of print]

Priyadarshini M, Kotlo KU, Dudeja PK, and Layden BT (2018) Role of short chain fatty acid receptors in intestinal physiology and pathophysiology. Compr Physiol 8 1091-1115.

Qin J, Li R, Raes J, Arumugam M, Burgdorf KS, Manichanh C, Nielsen T, Pons N, Levenez F, Yamada T, et al.; MetaHIT Consortium (2010) A human gut microbial gene catalogue established by metagenomic sequencing. Nature 464:59-65.

Quinn RA, Melnik AV, Vrbanac A, Fu T, Patras KA, Christy MP, Bodai Z, BeldaFerre P, Tripathi A, Chung LK, et al. (2020) Global chemical effects of the microbiome include new bile-acid conjugations. Nature 579:123-129.

Rachid S, Gerth K, Kochems I, and Müller R (2007) Deciphering regulatory mechanisms for secondary metabolite production in the myxobacterium Sorangium cellulosum So ce56. Mol Microbiol 63:1783-1796.

Ran L, Liu AB, Lee MJ, Xie P, Lin Y, and Yang CS (2019) Effects of antibiotics on degradation and bioavailability of different vitamin $\mathrm{E}$ forms in mice. Biofactors $\mathbf{4 5}$ : 450-462.

Roager HM and Licht TR (2018) Microbial tryptophan catabolites in health and disease. Nat Commun 9:3294.

Roager HM, Sulek K, Skov K, Frandsen HL, Smedsgaard J, Wilcks A, Skov TH, Villas-Boas SG, and Licht TR (2014) Lactobacillus acidophilus NCFM affects vitamin $\mathrm{E}$ acetate metabolism and intestinal bile acid signature in monocolonized mice. Gut Microbes 5:296-303.

Rogala AR, Oka A, and Sartor RB (2020) Strategies to dissect host-microbial immune interactions that determine mucosal homeostasis vs. intestinal inflammation in gnotobiotic mice. Front Immunol 11:214.

Rothhammer V, Mascanfroni ID, Bunse L, Takenaka MC, Kenison JE, Mayo L, Chao CC, Patel B, Yan R, Blain M, et al. (2016) Type I interferons and microbial metabolites of tryptophan modulate astrocyte activity and central nervous system inflammation via the aryl hydrocarbon receptor. Nat Med 22:586-597.

Russell WR, Labat A, Scobbie L, and Duncan SH (2007) Availability of blueberry phenolics for microbial metabolism in the colon and the potential inflammatory implications. Mol Nutr Food Res 51:726-731.

Saeui CT, Liu L, Urias E, Morrissette-McAlmon J, Bhattacharya R, and Yarema KJ (2018) Pharmacological, physiochemical, and drug-relevant biological properties of short chain fatty acid hexosamine analogues used in metabolic glycoengineering. Mol Pharm 15:705-720.

Saha S, Rajpal DK, and Brown JR (2016) Human microbial metabolites as a source of new drugs. Drug Discov Today 21:692-698.

Schoefer L, Mohan R, Schwiertz A, Braune A, and Blaut M (2003) Anaerobic degradation of flavonoids by Clostridium orbiscindens. Appl Environ Microbiol 69: $5849-5854$.

Sender R, Fuchs S, and Milo R (2016) Revised estimates for the number of human and bacteria cells in the body. PLoS Biol 14:e1002533.

Singh R, Chandrashekharappa S, Bodduluri SR, Baby BV, Hegde B, Kotla NG, Hiwale AA, Saiyed T, Patel P, Vijay-Kumar M, et al. (2019) Enhancement of the gut barrier integrity by a microbial metabolite through the Nrf2 pathway. Nat Commun 10:89.

Skelly AN, Sato Y, Kearney S, and Honda K (2019) Mining the microbiota for microbial and metabolite-based immunotherapies. Nat Rev Immunol 19:305-323.

Smith PM, Howitt MR, Panikov N, Michaud M, Gallini CA, Bohlooly-Y M, Glickman JN, and Garrett WS (2013) The microbial metabolites, short-chain fatty acids, regulate colonic Treg cell homeostasis. Science 341:569-573.

Sofyana NT, Zheng J, Manabe Y, Yamamoto Y, Kishino S, Ogawa J, and Sugawara T (2020) Gut microbial fatty acid metabolites (KetoA and KetoC) affect the progression of nonalcoholic steatohepatitis and reverse cholesterol transport metabolism in mouse model. Lipids 55:151-162.

Sonowal R, Swimm A, Sahoo A, Luo L, Matsunaga Y, Wu Z, Bhingarde JA, Ejzak EA Ranawade A, Qadota H, et al. (2017) Indoles from commensal bacteria extend healthspan. Proc Natl Acad Sci USA 114:E7506-E7515.

Stampfer L, Deutschmann A, Dür E, Eitelberger FG, Fürpass T, Gorkiewicz G, Heinz-Erian P, Heller I, Herzog K, Hopfer B, et al. (2017) Causes of hematochezia and hemorrhagic antibiotic-associated colitis in children and adolescents. Medicine (Baltimore) 96: $\mathrm{e} 7793$.

Steed AL, Christophi GP, Kaiko GE, Sun L, Goodwin VM, Jain U, Esaulova E, Artyomov MN, Morales DJ, Holtzman MJ, et al. (2017) The microbial metabolite desaminotyrosine protects from influenza through type I interferon. Science 357: 498-502.

Tang L, Qiu RG, Li Y, and Katz L (2003) Generation of novel epothilone analogs with cytotoxic activity by biotransformation. J Antibiot (Tokyo) 56:16-23.

Tang L, Shah S, Chung L, Carney J, Katz L, Khosla C, and Julien B (2000) Cloning and heterologous expression of the epothilone gene cluster. Science 287:640-642.

Tikhonova IG (2017) Application of GPCR structures for modelling of free fatty acid receptors. Handb Exp Pharmacol 236:57-77.

Torquato P, Marinelli R, Bartolini D, Giusepponi D, Cruciani G, Siragusa L, Galarini R, Sebastiani B, Gioiello A, and Galli F (2020) Vitamin E: metabolism and molecular aspects, in Molecular Nutrition (Patel VB ed) pp 487-518, Academic Press, Cambridge.

Tunaru S, Lättig J, Kero J, Krause G, and Offermanns S (2005) Characterization of determinants of ligand binding to the nicotinic acid receptor GPR109A (HM74A) PUMA-G). Mol Pharmacol 68:1271-1280.

van de Laar FA, Lucassen PL, Akkermans RP, van de Lisdonk EH, Rutten GE and van Weel C (2005) Alpha-glucosidase inhibitors for patients with type 2 diabetes: results from a Cochrane systematic review and meta-analysis. Diabetes Care 28:154-163.

van Duynhoven J, Vaughan EE, Jacobs DM, Kemperman RA, van Velzen EJJ, Gross G, Roger LC, Possemiers S, Smilde AK, Doré J, et al. (2011) Metabolic fate of polyphenols in the human superorganism. Proc Natl Acad Sci 108 (Suppl 1): 4531-4538.

Venkatesh M, Mukherjee S, Wang H, Li H, Sun K, Benechet AP, Qiu Z, Maher L, Redinbo MR, Phillips RS, et al. (2014) Symbiotic bacterial metabolites regulate gastrointestinal barrier function via the xenobiotic sensor PXR and Toll-like receptor 4. Immunity 41:296-310.

von Tesmar A, Hoffmann M, Abou Fayad A, Hüttel S, Schmitt V, Herrmann J, and Müller R (2018) Biosynthesis of the Klebsiella oxytoca pathogenicity factor tilivalline: heterologous expression, in vitro biosynthesis, and inhibitor development. ACS Chem Biol 13:812-819.

Vyhlídalová B, Krasulová K, Pečinková P, Marcalíková A, Vrzal R, Zemánková L, Vančo J, Trávníček Z, Vondráček J, Karasová M, et al. (2020) Gut microbial catabolites of tryptophan are ligands and agonists of the aryl hydrocarbon receptor: a detailed characterization. Int $J$ Mol Sci 21:2614.

Wang Y, Russo TA, Kwon O, Chanock S, Rumsey SC, and Levine M (1997) Ascorbate recycling in human neutrophils: induction by bacteria. Proc Natl Acad Sci USA 94: 13816-13819.

Windey K, De Preter V, and Verbeke K (2012) Relevance of protein fermentation to gut health. Mol Nutr Food Res 56:184-196.

Wlodarska M, Luo C, Kolde R, d'Hennezel E, Annand JW, Heim CE, Krastel P, Schmitt EK, Omar AS, Creasey EA, et al. (2017) Indoleacrylic acid produced by commensal Peptostreptococcus species suppresses inflammation. Cell Host Microbe 22:25-37.e6.

Wood J (2019) Bioinspiration in fashion-a review. Biomimetics (Basel) 4:16.

Yang X, Xie L, Li Y, and Wei C (2009) More than 9,000,000 unique genes in human gut bacterial community: estimating gene numbers inside a human body. PLoS One 4:e6074.

Zelante T, Iannitti RG, Cunha C, De Luca A, Giovannini G, Pieraccini G, Zecchi R D'Angelo C, Massi-Benedetti C, Fallarino F, et al. (2013) Tryptophan catabolites from microbiota engage aryl hydrocarbon receptor and balance mucosal reactivity via interleukin-22. Immunity 39:372-385.

Address correspondence to: Sridhar Mani, Albert Einstein College of Medicine, 1300 Morris Park Ave., Chanin 302D-1, Bronx, NY 10461. E-mail: sridhar.mani@einsteinmed.org; R. Balfour Sartor, Division of Gastroenterology and Hepatology, Department of Medicine, Center for Gastrointestinal Biology and Disease, University of North Carolina at Chapel Hill, Chapel Hill, NC 27599. E-mail: ryan_balfour_sartor@med.unc.edu; or Maayan Levy, Department of Microbiology, University of Pennsylvania, Philadelphia, PA 19104. E-mail: maayanle@pennmedicine.upenn.edu 